\title{
Memory deficits in Alzheimer's disease: The encoding hypothesis and cholinergic function
}

\author{
K. GEOFFREY WHITE and ANGELA C. RUSKE \\ University of Otago, Dunedin, New Zealand
}

\begin{abstract}
Forgetting functions generated by delayed matching-to-sample procedures allow delay-dependent effects to be distinguished from delay-independent effects on working memory. Parameters of negative exponential functions estimate initial discriminability (intercept) and rate of forgetting (slope). Forgetting functions for patients with Alzheimer's disease indicate that they differ from normal controls in terms of reduced initial discriminability - that is, in the encoding component of memory performancebut not convincingly in rate of forgetting. Reanalyses of previous studies with different species suggest that pro- and anticholinergic drugs influence initial discriminability in delayed matching-to-sampleperformance, but not rate of forgetting. The results of our reanalyses are consistent with the conclusion that the cholinergic system plays a role in the encoding component of working memory and that this is the main characteristic of the memory deficit shown by patients with Alzheimer's disease.
\end{abstract}

Alzheimer's disease (AD) is reported to affect at least $19 \%$ of individuals $75-84$ years old, and $47 \%$ of individuals over the age of 84 (Alzheimer's Association, 1996). Given the severe nature of the disease, the burden placed on caregivers, and the financial demands of the care of $\mathrm{AD}$ patients, there is growing interest in developing agents that not only reverse the cognitive deficits associated with AD, but also slow AD's progression. To date, however, there is only limited information about the aetiology and pathogenesis of AD (for a review, see Small, 1998), and this perhaps explains why only two drugs have been approved for the treatment of it.

AD can be definitely diagnosed only by means of histopathological examination of brain tissue following a patient's death (Small, 1998). In the early stages of the disease, impairment in short-term memory is apparent. As the disease progresses, patients are unable to perform many basic activities needed for everyday living. They may show decreased knowledge of current and recent events, impaired concentration, and decreased ability to travel, handle finances, or perform complex tasks. Pathologically, the classical hallmarks of AD include neuritic plaques, neurofibrillary tangles, and significant neuronal loss in the basal forebrain area (Bowen \& Davison, 1986). Various neurotransmitter systems may be affected, including the noradrenergic and serotonergic systems, but the involvement of the cholinergic system in AD has received the most attention. This is not surprising, given the marked impairment in memory performance following cholinergic system dysfunction. Over the last two decades, the hypothesis that the cholinergic system is the major neurotransmitter

Correspondence concerning this article should be sent to K. G. White, Department of Psychology, University of Otago, Dunedin, New Zealand (e-mail: kgwhite@otago.ac.nz,or ruske@world-net.co.nz). system involved in memory and learning has gained general acceptance (Bartus, 1978; Blokland, 1996; Everitt \& Robbins, 1997). The evidence reviewed here reinforces the suggestion that $\mathrm{AD}$ is related to cholinergic system dysfunction.

A recent comprehensive review by McDonald and Overmier (1998) describes a wide range of "animal models" for AD. Such models examine the effects of drugs such as scopolamine, of lesions (to the basal nucleus, medial septal area, fimbra/fornix, and hippocampus), and of betaamyloid administration, on performance in various delayed conditional-discrimination tasks in which subjects must rely on memory. The rationale for the "animal models" is that the effects of the drugs and lesions on task performance should resemble the deficits seen in AD patients. McDonald and Overmier suggest that for patients in the early stages of $\mathrm{AD}$, the deficit is manifest primarily as an increase in the rate of forgetting, whereas for patients in the late stages of the disease, the deficit is attentional in nature, and that deficits in encoding ability may be related to poor cognitive function. Below we question the evidence for this distinction, and suggest that the overall performance deficit shown by AD patients (at any stage) is best characterized as an encoding deficit, with little effect on rate of forgetting.

In the present brief review, we examine performance in the delayed matching-to-sample task, which includes a delay or retention interval between learning and remembering. As McDonald and Overmier (1998) suggest, tasks that include variation of the retention interval allow differentiation of the performance of AD patients from that of patients with other disorders, and, more importantly from our point of view, allow differentiation of the encoding and memorial components of performance. McDonald and Overmier emphasize the importance of distinguishing delay-dependent from delay-independent effects in de- 
layed conditional discrimination tasks and review evidence from animal models of AD in terms of that distinction. Here we emphasize the same distinction, but in addition show how a higher order analysis yields quantitative measures of the attentionalor encoding (delay-independent) versus memorial (delay-dependent) components of performance. First, we briefly describe the background to the cholinergic hypothesis and discuss the preclinical signs of $\mathrm{AD}$ as manifested in memory performance. Second, we describe the delayed matching-to-sample task and how higher order performance measures may be derived. Third, we show that the performance of $\mathrm{AD}$ patients in this procedure is best described in terms of an encoding deficit. Fourth, we review the results of previous studies, in which the delayed matching-to-sample performance of various species is influenced by drugs related to the cholinergic system in ways that resemble the deficits shown by AD patients.

\section{The Cholinergic Hypothesis}

The cholinergic system. The cholinergic system is made up of neurons that release the neurotransmitter acetylcholine (ACh). ACh receptors are found throughout the central nervous system and in high concentrations in the cerebral cortex, thalamus, and various nuclei in the basal forebrain. There are two main types of ACh receptors, muscarinic and nicotinic, so called because of their ability to bind muscarine and nicotine respectively. Five types of muscarinic receptors have been cloned to date, labeled M1-M5. The M1 receptor is found in abundance in the brain and has been associated with memory function because of the marked impairments in performance exhibited by laboratory animals when action at this receptor site is inhibited (Mash, Flynn, \& Potter, 1985; Perry, 1986; Whitehouse, 1986).

There are two principle components of the cholinergic hypothesis (Bartus, Dean, Beer, \& Lippa, 1982). First, forebrain cholinergic systems are vital for a variety of cognitive processes. Second, learning and memory deficits associated with aging may, in part, be attributed to a decline in performance of these forebrain cholinergic systems (Dunnett \& Fibiger, 1993). Earlier studies have shown that disruption to cholinergic function produced memory deficits which paralleled those seen in aged humans (Bartus, 1978; Drachman \& Leavitt, 1974).

Alzheimer's disease and brain function. The cholinergic hypothesis was later extended to include AD because of the decline of basal forebrain cholinergic neurons in the brains of AD sufferers (Coyle, Price, \& DeLong, 1983). Postmortem results show decreased choline acetyltransferase (ChAT) activity in the cerebral cortex and hippocampus of AD patients (Bartus et al., 1982; Volger, 1991). Further, the amount of ChAT decline has a reliable association with the severity of cognitive deficits seen in AD patients (Perry et al., 1978; Volger, 1991).

Pathological evidence from postmortem studies in AD patients suggests that the entorhinal cortex is one of the early sites of degeneration (Braak \& Braak, 1991, 1995; Mufsson et al., 1999). The entorhinal cortex connects the hippocampal formation with the neocortex, allowing the transfer of multimodal sensory information to the neocortex. Although hippocampal degeneration is seen in $\mathrm{AD}$, it is now believed that the entorhinal cortex is one of the sites first affected by the onset of AD. Magnetic resonance imaging (MRI) protocols also suggest that the entorhinal cortex is damaged early on in the course of AD. For example, de Toledo-Morrell, Goncharova, Dickerson, Wilson, and Bennett (2000) used quantitative structural MRI protocols to examine the effects of age on changes in entorhinal cortex volume. They compared entorhinal cortex and hippocampal volume in healthy elderly controls, patients with very mild $A D$, and elderly patients who were evaluated for cognitive complaints but did not meet criteria for dementia. The volumes of both entorhinal cortex and hippocampus in very mild AD and elderly patients with cognitive complaints were significantly less than in the control group. There was significantly more atrophy of the hippocampus in patients with mild AD. Interestingly, follow-ups of the elderly patients with cognitive complaints revealed that 12 of the 23 patients had developed $\mathrm{AD}$ and showed a further reduction in entorhinal volume.

Corkin (1998) used MRI scans to measure hippocampal volumes while subjects were performing a picture encoding and recognition task. Corkin was able to detect changes in the brain during the encoding and retrieval stages of the task in three groups of subjects: young normal subjects, older normal subjects, and subjects with AD. When all subjects performed a correct response, two changes were identified. First, the hippocampus was activated during the encoding of the picture, and second, the frontal lobe was activated during the recognition phase. Advanced age in the older normal and AD groups was associated with diminished activation in the hippocampus, consistent with the possibility of an impairment in their ability to encode information in comparison with their younger counterparts.

Preclinical diagnosis of AD. Recent reports have indicated the possibility of discriminating between presymptomatic $\mathrm{AD}$ and nondemented elderly patients on the basis of cognitive performance. Bäckman, Small, and Fratiglioni (2001) claimed that there is a long preclinical period (up to 6 years before diagnosis) during which episodic memory deficits are detectable. They reported an impairment in free recall and recognition performance in a group diagnosed as AD 6 years later, in comparison with normal aged matched controls. When these groups were retested 3 years after the first test, no further deterioration in recall or recognition was evident. Similarly, Chen et al. (2000) reported that delayed recall tests best discriminated between patients who would develop AD 1.5 years later and those who would remain nondemented.

These findings are important for two reasons. First, they indicate that early detection of AD is possible, allowing prevention and early intervention. Second, they 
show that the neurological changes present in the clinical diagnosis of $\mathrm{AD}$ (e.g., amyloid plaques and neurofibrillary tangles) accumulate at a relatively slow rate. They also support suggestions that early stages of the disease involve changes to the hippocampal and medial temporal lobe structures. This raises the question of the similarity between $\mathrm{AD}$ and medial temporal lobe amnesia.

Temporal lobe amnesia. Medial temporal lobe amnesia results from bilateral damage to the hippocampus and adjacent structures - in particular, the entorhinal, perirhinal, and parahippocampal cortex (Squire \& Zola-Morgan, 1991). The most studied patient with medial temporal lobe amnesia is H.M. Although initial studies suggested that the main deficit seen in H.M. was an abnormally fast rate of memory decay, it is now believed that that finding may have been an artifact of the procedure used to test memory performance (McKee \& Squire, 1992). The earlier studies (Huppert \& Piercy, 1978, 1979) had required H.M. to make a forced-choice yes/no recognition response for pictures shown during the sample phase. Whereas retention was normal for a short 10-min retention interval, H.M. performed more poorly than normal subjects on the longer retention intervals of 1 and 7 days. Freed, Corkin, and Cohen (1987) used a procedure very similar to that used by Huppert and Piercy. But by allowing H.M. to see old and new items side by side, H.M. performed normally at 1 and 7 days. Further, Freed and Corkin (1988) found that H.M. could retain visual information at a level similar to that of a normal subject, for up to 6 months. McKee and Squire (1992) have also found that amnesic subjects with temporal lobe damage can perform similarly to normal subjects on memory tasks with delays of about $30 \mathrm{~h}$.

In the studies above, amnesic patients were given considerably more time to study each item to be remembered, ranging from 8 to $20 \mathrm{sec}$ per item, whereas the normal subjects were given $1 \mathrm{sec}$. That is, more time was needed for amnesic subjects to encode information. The performance of amnesic subjects on memory tasks seems to mimic the performance of patients in the early stages of AD. The behavioral and functional similarities between $\mathrm{AD}$ and amnesia are consistent with the involvement of the entorhinal cortex and hippocampus in the encoding process (de Toledo-Morrell et al., 2000).

\section{Delayed Matching to Sample}

Of the different behavioral procedures that have been employed to investigate cognitive deficits associated with cholinergic system dysfunction, one procedure that has been very popular is delayed matching-to-sample(DMTS). First, it allows direct comparison of different techniques used for manipulating cholinergic function, and comparison of the behavioral effects of these manipulations across species, including humans. Second, the data generate forgetting functions that can be described very well by negative exponential equations (White, 1985, 2001) or other negatively accelerated functions (White \& Harper, 1996; Wixted \& Ebbesen, 1991). The advantage of such equations is that the two parameters provide higher order indices of remembering. Thus, factors that affect the encoding of information, such as attention or perception, can be assessed independently of changes that affect the retrieval of information. This will be discussed more fully below.

The DMTS procedure is a type of discrete-trials conditional discrimination (White \& Alsop, 1993). It involves the presentation of a sample stimulus on each trial (e.g., a red or a green hue) which is withdrawn after a fixed number of observing responses or after a fixed time. The offset of the sample stimulus is immediately followed by a retention interval with a duration typically varying between 0 and $30 \mathrm{sec}$. When animals are used as subjects, there is no opportunity to respond during the retention interval. At the completion of the retention interval, the choice stimuli (e.g., red and green) are presented and the subject is rewarded for correctly choosing the stimulus presented during the sample phase. There are several variations of the standard DMTS procedure, including delayed nonmatchingto-sample, where the correct choice is the stimulus that does not match the sample; delayed symbolic matchingto-sample, where the sample and choice stimuli are not identical; and delayed matching-to-position, where the sample stimuli are locations.

Procedures in which the four or five retention interval durations are varied within a session have several advantages over procedures in which the retention interval is held constant (White \& Bunnell-McKenzie, 1985). First, the short intervals help to maintain attention to the task and minimize response bias. Second, when a major independent variable other than retention interval duration is introduced (e.g., the administration of drugs), there are none of the order effects that would otherwise occur when different retention intervals were varied over conditions. Third, the data can be plotted as a forgetting function that is amenable to a quantitative analysis.

The typical measure of performance in DMTS is the proportion of correct matching responses at each retention interval. When proportion correct is plotted against retention interval duration, the negatively accelerated curve describes a forgetting function. Although proportion correct is easily interpreted and is traditionally used, it has two problems. The first is that it is confounded with response bias and therefore does not provide a true measure of memorability (McCarthy \& White, 1987). The second is that it is bounded at chance (.5 in a two-choice experiment) and 1.0. That is, if performance is high overall, a general ceiling effect will mask the possible influence of an independent variable (such as drug administration). Similarly, inaccurate performangce near chance level will be insensitive to the influence of an independent variable. In effect, these ceiling and floor effects are the result of the unequal-interval scale for proportion correct. These effects are precluded by a ratio-based measure of discriminability that varies on an equal-interval scale, assuming a linear relation between the discriminability measure and the underlying theoretical process (Loftus, 1978). One fa- 
miliar measure is discriminability, $d^{\prime}$, derived from signal detection theory. Another measure of discriminabilityis $\ln \alpha$, from choice theory (Luce, 1963) or log $d$ of behavioral detection theory (Davison \& Tustin, 1978). These measures are the same, except for the base for the logarithm (natural vs. base 10). They are linearly related to $d^{\prime}$. The discriminability measures assume that true memorability is assessed independently of response bias.

The decrement in performance with increasing retentioninterval duration is clearer for discriminability measures, owing to the absence of ceiling and floor effects. The measure from choice theory or behavioral detection theory is easily calculated (Macmillan \& Creelman, 1991). For correct and error responses following Sample $1\left(c_{1}\right.$ and $\left.e_{1}\right)$ and for correct and error responses following Sample 2 $\left(c_{2}\right.$ and $\left.e_{2}\right)$, discriminability is

$$
\log d=0.5 \log \left[\left(c_{1} / e_{1}\right)\left(c_{2} / e_{2}\right)\right] .
$$

If at least four retention intervals are used, it is possible to fit a function to the data by using a nonlinear least squares method. White (1985) found that a simple negative exponential function, $y=a \cdot \exp (-b \cdot t)$, provides a satisfactory fit to a wide range of data from DMTS procedures. Forgetting functions from this and other procedures tend to be better fit by the power function or an exponential function with time scaled as $\sqrt{t}$ (White, 2001; White \& Harper, 1996; Wixted \& Ebbesen, 1991). The function fitted to data reanalyzed in the present paper is $y=a$. $\exp (-b \cdot \sqrt{t})$, which is among the best-fitting functions for a wide range of data (Rubin \& Wenzel, 1996).

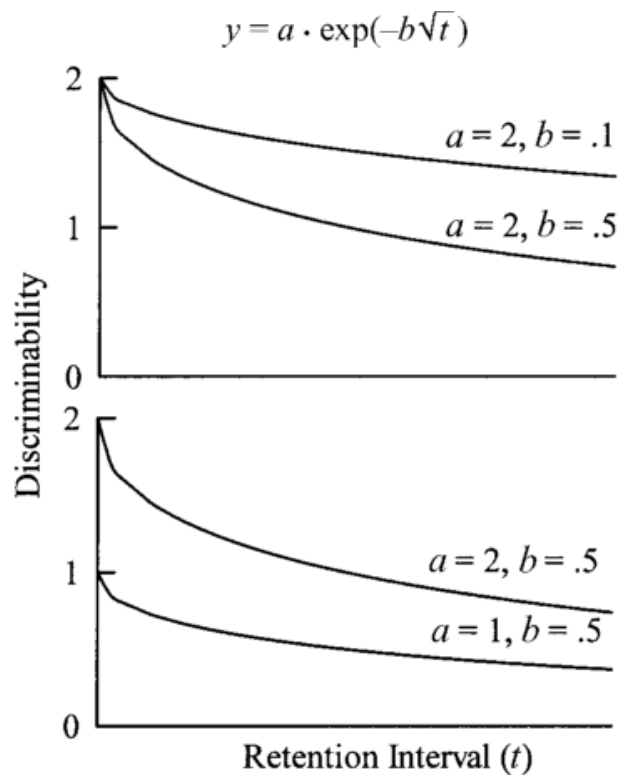

Figure 1. Negative exponential forgetting functions with time scaled as $\sqrt{t}$ which differ in rate of forgetting (slope, parameter $b$ ) but not initial discriminability (intercept, parameter $a$ ), or initial discriminability but not rate of forgetting.
The $y$-intercept, $a$, describes performance in the absence of a memory requirement and can be defined as initial discriminability. The parameter $b$ describes the rate of decrement in matching accuracy or discriminability with increasing retention interval duration and can be defined as rate of forgetting. Figure 1 illustrates how forgetting functions can differ in their intercept or initial discriminability, or in their rate of forgetting. Because forgetting functions vary over a wide range of procedures, in both the overall level of performance and the way that performance changes with increasing retention interval duration (White, 1985), it is useful to summarize the whole function in terms of the parameters of a fitted function, especially when one is attempting to determine the effects of drugs on overall performance.

Most importantly, the slope parameter of the fitted function allows comparison of different rates of forgetting. In many cases, differences in rates of forgetting are evaluated in terms of interactions between condition and delay in analysis of variance. This treatment is associated with a number of problems (Loftus, 1978, 1985), one of which concerns the nature of the scale that is used. The best method is to compare the slope parameter of fitted functions (White, 1985; Wixted, 1990), especially when performance measures vary on scales that are theoretically equal-interval, such as $d^{\prime}$ or $\log d$.

When the exponential function is fitted to data from DMTS procedures in which the various independent variables described above have been manipulated, values for the parameters of the fitted functions vary systematically (White, 1991, 2001). Generally, changes in $a$ are associated with encoding operations and are influenced by factors expected to alter attention to the sample stimulus (White, 1985). For example, in delayed matching-to-sample in humans, increasing the sample set size decreases initial discriminability, $a$, without affecting rate of forgetting (Adamson, Foster, \& McEwan, 2000). Increasing the number of observing responses to the sample in pigeons increases initial discriminability without affecting rate of forgetting (White, 1985). Rate of forgetting, described by parameter $b$, is influenced by factors expected to be related to retrieval. For example, if the conditions for retrieval are rendered more difficult, such as the introduction of retroactive interference, the rate of forgetting increases (Harper $\&$ White, 1997). Similarly, proactive interference from having to remember a different item on the prior trial results in an increase in the rate of forgetting on the current trial (Edhouse \& White, 1988). Because the memorial aspects of the task are associated with rate of forgetting, it is of interest to ask whether the parameter $b$ changes with drugs that are meant to influence memory systems.

\section{DMTS Performance of AD Patients}

Money, Kirk, and McNaughton (1992) compared patients clinically diagnosed with AD with a group of agematched control subjects in a DMTS procedure. With delays ranging from 0 to $32 \mathrm{sec}$, participants were required 
Money, Kirk, \& McNaughton (1992)

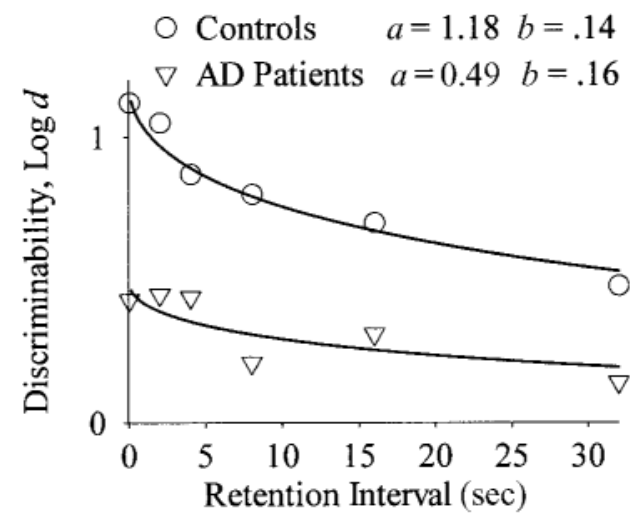

Sahgal et al. (1992)

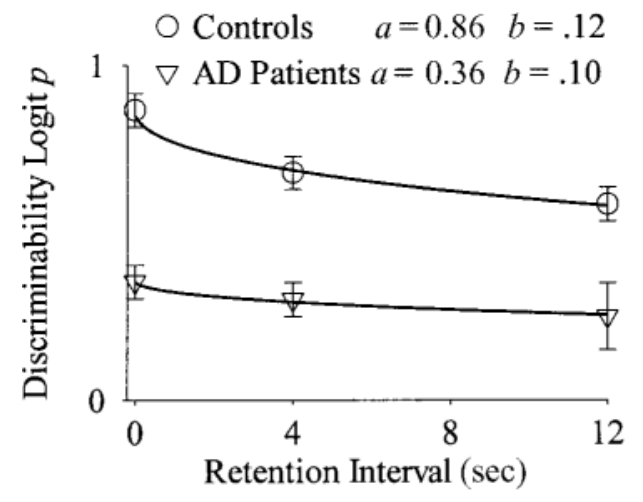

Kopelman (1985, Experiment 2)

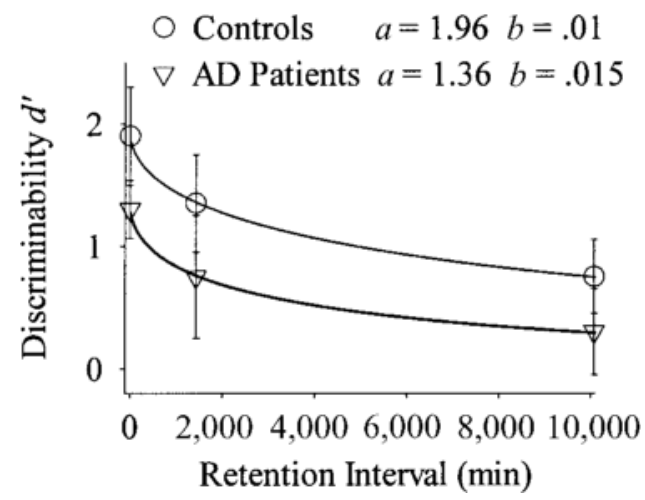

Figure 2. Forgetting functions from delayed matching-to-sample procedures for control subjects and $A D$ patients from three studies. Best-fitting functions for $y=a \exp (-b \sqrt{t})$ and their parameter values $(a, b)$ are shown. In most cases, the best-fitting functions accounted for about $99 \%$ of the variance in the data.

to match circle diameter sizes presented during the sample phase. AD patients performed less accurately overall than the age-matched control subjects. When we fitted exponential functions with time scaled as $\sqrt{t}$ to their data, the intercept parameter $a$ was lower for the AD patients than for the controls, whereas there was no difference in the value of the $b$ parameter. That is, encoding processes were impaired in the AD patients, but not rate of forgetting. Money et al. arrived at the same conclusion on the basis of fitting simple exponential functions to their data, taking into account the longer response latencies of the $\mathrm{AD}$ patients. Figure 2 shows the results of our reanalysis, along with the reanalysis of results reported by Sahgal et al. (1992) and Kopelman (1985). All three studies show a similar pattern, with the forgetting functions for $\mathrm{AD}$ patients having lower initial discriminability than those for controls, but with no differences in the rate of forgetting.

Sahgal et al. (1992) compared the matching ability of patients diagnosed with senile dementia of the Alzheimer's type and senile dementia of the Lewy Body type (SDLT) and that of age-matched control subjects in computergenerated simultaneous matching-to-sample (SMTS) and DMTS procedures. They found that both senile dementia groups performed significantly less accurately in the DMTS task than did the control subjects. Similarly, both dementia groups were significantly less accurate in SMTS, although subjects suffering from SDLT performed less accurately than $\mathrm{AD}$ patients. Because performance was low overall, even at the shortest retention interval of $0 \mathrm{sec}$, and also in SMTS, the authors suggested that encoding mechanisms were affected. In this and in other studies described below where proportion correct was used as the dependent measure, we reanalyzed the data, by transforming proportion correct $(p)$ to $\operatorname{logit}(p)=\log (p / 1-$ $p$ ) in order to plot the data on a scale that has properties similar to those of the scale for $d^{\prime}$ and $\log d$. When there is no response bias, a safe assumption for group data, logit $p=\log d$, the discriminability measure used in our own studies. When we fitted negative exponential functions for $\sqrt{t}$ to the logit $p$ transforms of the data reported by Sahgal et al., we confirmed that the performance of the SDLT and $\mathrm{AD}$ groups was characterized by a reduction in initial discriminability $(a)$, without a change in the rate of forgetting $(b)$.

Kopelman (1985) compared the ability of subjects diagnosed with $\mathrm{AD}$ and that of age-matched control subjects in a procedure in which subjects recalled words after delays ranging from 0 to $20 \mathrm{sec}$. AD patients performed less accurately than control subjects at all delays. Reanalysis of their data was not straightforward, however, because a discriminability measure could not be calculated and the control subjects were at ceiling at zero delay. In their second experiment, Kopelman used Huppert and Piercy's $(1978,1979)$ picture recognition task and reported $d^{\prime}$ discriminability measures. When we reanalyzed their data by fitting negative exponential functions with time scaled as $\sqrt{t}$ to the data points, an encoding deficit was evident, as is shown by a reduction in the initial discriminability parameter (Figure 2). Kopelman concluded that the deficit in AD patients is "primarily an acquisition or learning deficit, [and that] once information is learned to a criterion, it is forgotten at a normal rate" (p. 635). 
The studies above included AD patients in the later stages of the disease. McDonald and Overmier (1998) have also concluded that "Alzheimer patients in the more advanced stages of the disease ... show equal impairments at all delays [of a delayed conditional discrimination task], i.e., the slope is equal to that of controls, but the $y$-intercept drops considerably. This situation may be indicative of a perceptual, encoding, or attentional deficit" (p. 105). McDonald and Overmier, however, concluded that patients in the early stages of AD show faster rates of forgetting relative to controls.

We were unable to confirm the latter conclusion on the basis of the studies that were said to support it. For example, in the studies by Flicker and others with patients in early stages of AD (Flicker, Ferris, Kalkstein, \& Serby, 1994; Flicker, Ferris, \& Reisberg, 1991), there was not always an appropriate control group and delays were not varied in such a way as to distinguish between initial discriminability and rate of forgetting. The results of one study appear to allow the appropriate analyses (Sahakian et al., 1988). This study included a DMTS test with early-stage $\mathrm{AD}$ patients and controls. Our reanalysis of the proportion correct data presented in their Figure 3 gave initial discriminability values of .63 and .96 for the $\mathrm{AD}$ patients and controls, respectively, and rates of forgetting of 0.18 and 0.01 . The initial discriminability value was clearly lower for the AD patients, but the difference in rate of forgetting is difficult to interpret because the retention intervals employed (ranging from 0 to $16 \mathrm{sec}$ ) were confounded with the order of their presentation in the test. A further problem was that the variance in the data, especially for the control group, was sufficiently large that the rate of forgetting would not be significantly different from the rate of forgetting for the SDLT patients. When we transformed percent correct to logit $p$ to produce an equal-interval scale, the large variance in the control group precluded satisfactory fits of the exponential function. The only clear conclusion that can be drawn from the study by Sahakian et al., therefore, is that AD patients in the early stages of the disease show a deficit in encoding processes in the same way that patients in the later stages do. Evidence for differences in rate of forgetting is equivocal.

One further study that claimed an increased rate of forgetting in early-stage AD patients was reported by Hart, Kwentus, Harkins, and Taylor (1988). Several procedural problems, however, compromise the conclusion from this study. As it happens, AD patients and controls showed the same rate of forgetting from $10 \mathrm{~min}$ to $2 \mathrm{~h}$ in a picture recognition test. At the third interval studied, $48 \mathrm{~h}$, the $\mathrm{AD}$ patients improved their performance and the controls worsened. The recognition tests, however, were repetitions, and additionally, the AD patients clearly had difficulty learning the material at the outset.

Recognition versus recall. The DMTS procedure is a recognition procedure, in that the comparison that matches the sample stimulus is chosen at the time of retrieval. Several studies indicate that, at least for amnesics, there is no difference in the rate of forgetting relative to that of controls for both recognition and recall procedures. Perhaps the most convincing evidence was reported by Haist, Shimamura, and Squire (1992). Haist et al. tested amnesic patients at retention intervals ranging from $15 \mathrm{sec}$ to 1 day for recall and $15 \mathrm{sec}$ to 2 weeks for recognition. Control subjects were tested at retention intervals ranging from $15 \mathrm{sec}$ to 8 weeks for both recall and recognition. When the forgetting functions for recall and recognition were plotted on axes that allowed them to overlap, the data clearly showed no difference in rate of forgetting for both recall and recognition.

More recently, Isaac and Mayes (1999) claimed that rate of forgetting is greater for amnesic patients than for controls when these subjects are tested for recall of semantically organized word lists. In contrast, rate of forgetting for free recall of unrelated words, and for recognition, did not differ for amnesics and controls. Their result for recall of organized lists depended on the significant interaction resulting from the reduction in recall scores from 20 -sec to 3 -min retention intervals of 6.6 to 2.8 items for amnesics, in comparison with the increase from 8.9 items to 9.5 items for controls. A major difficulty of interpretation of this interaction concerns the issue of scaling, raised by Loftus (1978). Specifically, control performance was at a high level overall, allowing little opportunity for retention-interval duration to influence performance. Isaac and Mayes dealt with this problem in their second experiment by arranging similar performance levels for amnesics and controls. For recall of organized lists, recall scores at 20-sec and 3-min retention intervals were 6.9 and 2.3 for amnesics, and 6.0 and 4.2 for controls. A "close to significance" interaction of group, delay, and condition (including unrelated word lists) justified a two-way analysis of variance on the data for organized lists. This showed a significant group $\times$ delay interaction, thus providing evidence for the possibility of faster rate of forgetting of semantically organized lists in amnesics. For all other cuedrecall and recognition conditions reported by Isaac and Mayes, there was no evidence for differences in rate of forgetting for amnesics versus controls. It is therefore difficult to conclude with any confidence that amnesics might show greater rate of forgetting than controls on recall but not on recognition tasks. A further difficulty is that Isaac and Mayes used only two retention intervals (unlike the research of Squire and his colleagues, where many intervals are studied). As emphasized by Wixted (1990), the appropriate way of estimating rate of forgetting is not through interactions with delay in analysis of variance, but from the rate parameter of fitted functions. With only two retention intervals, fitting functions is precluded. Before it can be concluded with any confidence that amnesics show a greater rate of forgetting in their recall of semantically related word lists, the study by Isaac and Mayes should be replicated with a wide range of retention intervals.

\section{Cholinergic System Function and DMTS}

The present selective review summarizes some of the studies using the DMTS procedure to examine the effects of drugs that are presumed to inhibit or enhance choliner- 
Table 1

Summary of Studies Using DMTS Procedures That Show Deficits in Initial Discriminability Following Cholinergic System Dysfunction

\begin{tabular}{lll}
\hline \multicolumn{1}{c}{ Author } & Species & \multicolumn{1}{c}{ Manipulation } \\
\hline Bartus (1978) & Monkeys & Scopolamine \\
Bartus \& Johnson (1976) & Monkeys & Scopolamine \\
Dawson \& Iversen (1993) & Rats & Scopolamine \\
Dunnett (1985) & Rats & Scopolamine \\
Dunnett, Rogers, \& Jones (1989) & Rats & IBO lesions \\
Kirk, White, \& McNaughton (1988) & Rats & Scopolamine \\
Kopelman (1985) & Humans & AD patients \\
Miyamoto et al. (1996) & Rats & Scopolamine \\
Money, Kirk, \& McNaughton (1992) & Humans & AD patients \\
Penetar \& McDonough(1983) & Monkeys & Atropine \\
Robinson, Wenk, Wiley, Lappi, \& Crawley (1996) & Rats & SAP-192, IBO lesions \\
Ruske, Fisher, \& White (1997) & Pigeons & Scopolamine \\
Ruske \& White (1999) & Pigeons & Scopolamine \\
Safer \& Allen (1971) & Humans & Scopolamine \\
Sahgal et al. (1992) & Humans & AD patients \\
Savage \& Parsons (1997) & Rats & Scopolamine \\
Teal \& Evans (1982) & Pigeons & Scopolamine \\
\hline
\end{tabular}

gic function. We include studies in which the retention interval was varied over several values, thus allowing a quantitative analysis. Table 1 lists studies that examined the effects of cholinergic antagonists, each of which is assumed to affect the action of a particular receptor, and Table 2 lists studies that examined the effects of cholinergic agonists. The main species used in the various studies included pigeons, rats, and monkeys. If DMTS performance is to be influenced by drug administration in a way that resembles the performance impairment shown by AD patients, we would expect to see changes in initial discriminability but not rate of forgetting. For the reanalyses reported below, the equation $y=a \cdot \exp (-b \cdot \sqrt{t})$ was fitted to discriminability measures or to the logit transforms of the percent correct measures reported in the original papers in order to achieve equal interval scales. In most cases, variance accounted for by the best-fitting function was better than $90 \%$. Because of the large number of conditions reported, parameter values are not given in Tables 1 and 2, but the pattern of results was very similar across all studies. Figure 3 shows examples in which the frequently used cholinergic antagonist scopolamine reduces initial discriminability without affecting rate of forgetting, as well as examples in which cholinergic agonists reversed the effect of scopolamine by increasing initial discriminability.

Pigeons. The cholinergic system in the pigeon brain appears to be similar to that in mammals (Medina \&
Reiner, 1994). Teal and Evans (1982) investigated the effects of three doses of scopolamine $(0.01-0.1 \mathrm{mg} / \mathrm{kg}$ ) on matching performance in pigeons. Scopolamine produced a dose-related decrease in performance. Our reanalysis (White, Ruske, \& Colombo, 1996) showed that this deficit was due to a decrease in the encoding component of performance. A similar effect on pigeons' performance following scopolamine administration observed by Ruske, Fisher, and White (1997) is replotted in the left middle panel of Figure 3 (also see Ruske \& White, 1999). Figure 3 shows a marked decrease in discriminability $(\log d)$ following the administration of $0.03 \mathrm{mg} / \mathrm{kg}$ of scopolamine. The parameter values of the fitted exponential functions demonstrated that this change occurred in initial discriminability and not in rate of forgetting. In the same study (Ruske et al., 1997), we administered AF150(s), a novel agonist that has been shown to affect the action of the M1 muscarinic receptor in the cholinergic system. Figure 3 shows the results of administration of $4 \mathrm{mg} / \mathrm{kg}$ AF150(s) with continued administration of scopolamine. The agonist partially reversed the encoding deficit associated with scopolamine administration. Again, this change was reflected in an increase in initial discriminability of the forgetting functions, without any significant change in rate of forgetting.

Parkes and White (2000) reported the same reduction in initial discriminability with scopolamine administration

Table 2

Summary of Studies Using DMTS Procedures That Showed Improvements in Initial Discriminability Following the Administration of Procholinergic Agents

\begin{tabular}{llll}
\hline \multicolumn{1}{c}{ Author } & Species & \multicolumn{1}{c}{ Drug Name } & Receptor Type \\
\hline Dawson et al. (1991) & Rats & Physostigmine & ACh Inhibitor \\
Dawson \& Iversen (1993) & Rats & Eptastigmine & ACh Inhibitor \\
Dawson \& Iversen (1993) & Rats & E2020 & ACh Inhibitor \\
Dunnett (1985) & Rats & Physostigmine & ACh Inhibitor \\
Miyamoto et al. (1996) & Rats & TAK-147 & ACh Inhibitor \\
Ruske, Fisher, \& White (1997) & Pigeons & AF150(s) & M1 agonist \\
\hline
\end{tabular}


Bartus (1978)

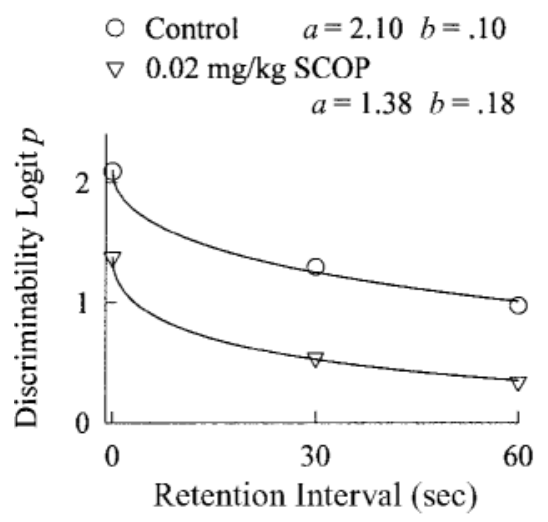

Ruske, Fisher, \& White (1997)

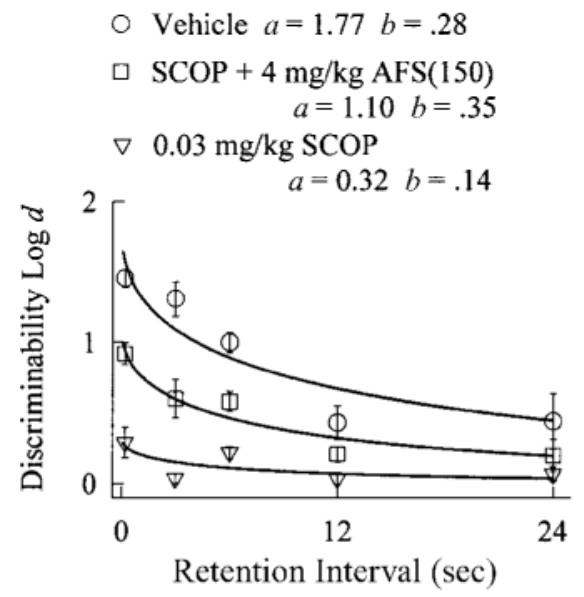

Dawson \& Iversen (1993)

$$
\begin{aligned}
& \circ \text { Vehicle } a=1.88 b=.11 \\
& \nabla \text { SCOP }+1 \mathrm{mg} / \mathrm{kg} \text { L-687,306 } \\
& a=0.65 \quad b=.21 \\
& \text { Scopolamine } a=0.22 \quad b=.21
\end{aligned}
$$

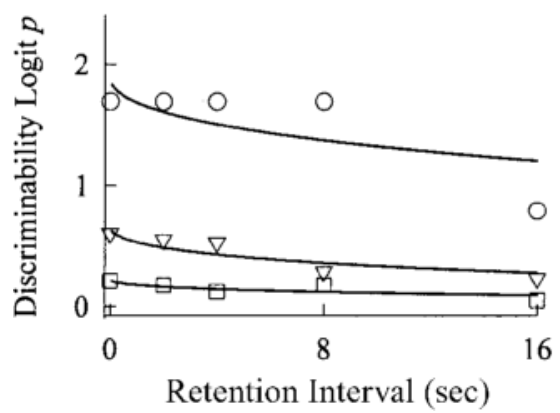

Parkes \& White (2000)

$\circ$ Vehicle $\quad a=1.62 \quad b=.31$
ㅁ.03 mg/kg SCOP $a=.19 \quad b=.25$

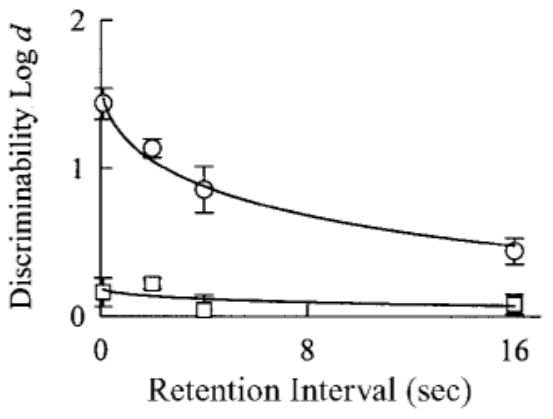

Teal \& Evans (1982)

$\circ$ Vehicle $\quad a=1.70 b=.29$

$\nabla .01 \mathrm{mg} / \mathrm{kg} \mathrm{SCOP} a=1.14 b=.23$

ㅁ. $.03 \mathrm{mg} / \mathrm{kg}$ SCOP $a=0.61 b=.34$

$\diamond .10 \mathrm{mg} / \mathrm{kg} \mathrm{SCOP} a=0.27 b=.26$

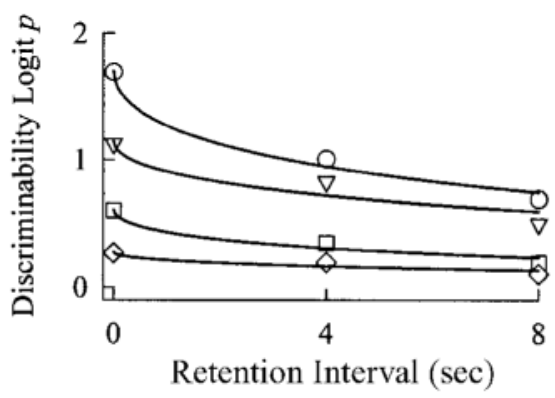

Dunnett (1985)

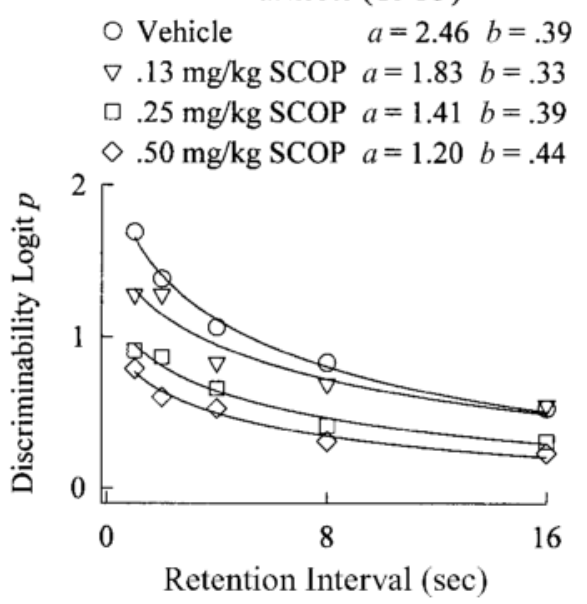

Figure 3. Forgetting functions for monkeys (top left), pigeons (top right and middle) and rats (bottom) in delayed matching-to-sample procedures. In each study, administration of the cholinergic antagonist scopolamine produced a reduction in the intercept $a$ of the best-fitting negative exponential function. In the studies by Ruske, Fisher, and White (1997) and by Dawson and Iversen (1993), the cholinergic agonists (acting on the muscarinic receptors) AF150(s) and L-687, 306 in part reversed the scopolamine deficit by increasing initial discriminability $(a)$. 
for DMTS performance in pigeons as that shown in the previous studies. Parkes and White demonstrated that the scopolamine-induced deficit could be reversed by administration of glucose, which facilitates release of acetylcholine. A novel aspect of their study was the glucoseinduced reversal of a performance deficit in initial discriminability, created by reducing the observing-response requirement to the sample. This manipulation validated the claim that initial discriminability reflects the encoding component of memory performance (White, 1985).

Rats. Dunnett (1985) administered four dose levels of scopolamine to rats performing a delayed matching-toposition task. The dose-related decrease in matching accuracy was obvious at short retention intervals. Similar encoding deficits were also observed following ibotenic acid (IBO) lesions of the nucleus basalis. Physostigmine, an AChE inhibitor, significantly improved performance at all delays. Our reanalysis of their data showed that the physostigmine improvement was reflected in an increase in initial discriminability. Kirk, White, and McNaughton(1988) administered five dose levels of scopolamine (ranging from 0.005 to $0.375 \mathrm{mg} / \mathrm{kg}$ ) to rats performing a delayed conditional discrimination task. They reported significant changes in initial discriminability parameters of the fitted exponential functions, suggesting attentional or encoding deficits, without significant changes in rate of forgetting.

Dawson and Iversen (1993) compared the ability of five procholinergic drugs (L-689, 660; AF102B; L-687, 306; E2020; and eptastigmine) to reverse scopolamine-induced deficits in rats under a variety of different behavioral procedures, including delayed matching-to-position. Consistent with earlier research, scopolamine significantly reduced accuracy across all retention intervals. By fitting the simple exponential equation to logit $p$ transformations of their data, we confirmed an encoding deficit. Interestingly, only the two AChE inhibitors (E2020 and eptastigmine) were capable of partially reversing the scopolamineinduced deficit, an improvement that appeared to be due to an increase in the encoding component of performance. Figure 3 (left bottom panel) shows the results of adding the muscarinic agonist L-687, 306 to scopolamine (Dawson \& Iverson, 1993). As for the result of adding AF150(s) to scopolamine (Figure 3, left middle panel), L-687, 306 partly reversed the scopolamine deficit by increasing initial discriminability.

Robinson, Wenk, Wiley, Lappi, and Crawley (1996) compared the effects of IBO lesions to the nucleus basalis and medial septum with the selective cholinergic neurotoxin ${ }^{192} \mathrm{IgG}$-Saporin (192-SAP) in rats. They reported delay-independent changes in delayed nonmatching-toposition following both 192-SAP administration and IBO lesions. Our reanalysis showed a reduction in initial discriminability following both lesion types.

Miyamoto et al. (1996) compared the ability of two AChE inhibitors, TAK-147 and tacrine, to reverse DMTP deficits in rats following scopolamine administration. They noted that the primary symptom associated with scopolamine administration was an attentional deficit. Our reanalysis confirmed this. They found that TAK-147 was capable of partially ameliorating the deficit associated with scopolamine administration, but were not able to detect any improvement in performance following tacrine administration. Our reanalysis showed that improved performance was due to an increase in initial discriminability, with no apparent change in rate of forgetting.

More recently, Savage and Parsons (1997) compared the effects of scopolamine and the NMDA receptor antagonist MK-801 on delayed matching-to-position in rats. Despite claims of delay-dependent changes following the administration of both of these drugs, impaired performance across all delays was evident, particularly at the $0.5-\mathrm{mg} / \mathrm{kg}$ dose level of scopolamine. Our reanalysis again showed a change in initial discriminability but not rate of forgetting, indicative of an effect on the encoding component of performance.

Monkeys. The results of some of the earlier studies of scopolamine administration in monkeys were interpreted as memorial effects. However, our reanalyses challenge this claim. For example, Bartus (1978) administered dose levels of scopolamine ranging from 0.015 to $0.02 \mathrm{mg} / \mathrm{kg}$ to monkeys in a computerized DMTS procedure. Even at the short retention interval of $0 \mathrm{sec}$, there was some evidence of impaired matching accuracy. Consistent with other studies, our reanalysis suggested that initial discriminability and not rate of forgetting was affected by scopolamine administration (Figure 3, left top panel). Interestingly, low dose levels had been selected to avoid the more general, nonmnemonic effects thought to occur with higher dose levels. But even very low doses of scopolamine reduce initial discriminability without affecting rate of forgetting (Kirk et al., 1988).

Bartus and Johnson (1976) tested the effects of two dose levels of scopolamine $(0.015$ and $0.03 \mathrm{mg} / \mathrm{kg})$ in monkeys in a DMTS task. In comparison with controls, all 3 monkeys performed poorly at all retention intervals (ranging from 0 to $10 \mathrm{sec}$ ). Despite suggestions that the impairment in performance was most likely due to memoryrelated deficits, our reanalysis suggests that an encoding deficit was apparent. Interestingly, 2 of the 3 monkeys performed below $100 \%$ in a simultaneous matching-tosample procedure, where the sample stimulus remained on during the choice phase. This perhaps also suggests that the monkeys' ability to attend to or encode the information was somewhat impaired by scopolamine.

Penetar and McDonough (1983) administered two anticholinergic agents, atropine and benactyzine, as well as the AChE inhibitor physostigmine, to monkeys performing a DMTS task. Consistent with the other studies reviewed in this paper, both anticholinergic agents resulted in disrupted accuracy across all retention intervals tested $(0-$ $16 \mathrm{sec})$. Impaired accuracy was apparent at the 0 -sec retention interval at 0.14 - and $0.44-\mathrm{mg} / \mathrm{kg}$ dose levels of atropine, and $0.182-$ and $1.82-\mathrm{mg} / \mathrm{kg}$ dose levels of benactyzine. The higher order parameters derived from our 
exponential fits to logit $p$ transforms of their data suggest the occurrence of encoding rather than retrieval deficits. Physostigmine administered on its own did not significantly alter performance at any dose level tested.

Humans. The disruption in the attentional or encoding component of memory performance has also been found in humans when cholinergic function has been impaired by drug administration. For example, Safer and Allen (1971) administered scopolamine to young healthy adults and tested their ability to recall digits over retention intervals ranging from 0 to $20 \mathrm{sec}$. Our reanalysis revealed that scopolamine administration markedly decreased initial discriminability in comparison with baseline control levels. There was no change in rate of forgetting.

\section{Conclusion}

The primary advantage in generating a forgetting function across a range of different retention intervals is that delay-dependent effects can be distinguished from delayindependent effects. By fitting a function to the data such as the negative exponential, one can estimate values for two parameters. One of them describes the intercept or initial discriminability; the other, the rate of forgetting. The two parameters of forgetting functions capture the important processes of memory performance: encoding and retrieval. Forgetting functions for AD patients indicate that they differ from those for normal controls in terms of the initial discriminability parameter. For patients in both the early and late stages of the disease, it therefore seems that the main contribution to their memory deficit is an impairment in the attentional or encoding component of performance.

Several factors may contribute to the level of initial discriminability in working memory procedures. If, for $\mathrm{AD}$ patients, the rules of the task are incompletely learned, the overall level of performance will be low, reflected in a low value of initial discriminability. Although AD patients may have initial difficulty in learning the task, typically they remember the rules or procedural requirements (McDonald \& Overmier, 1998). Instead, their difficulty stems from aspects of the task that change from trial to trial. Perceptual disparity of the stimuli to be remembered influences the level of initial discriminability, but this is assumed to be the same for AD patients and controls. Successful encoding requires attention to the stimuli to be remembered on a given trial. Although $\mathrm{AD}$ patients may be capable of attending to the stimuli, occasional lapses of attention are sufficient to disrupt encoding and reduce initial discriminability. The attentional component therefore seems the most likely candidate to explain the encoding difficulty experienced by AD patients. This conclusion is consistent with conclusions from previous research that the primary symptom associated with $\mathrm{AD}$ is due to attentional deficits (Grady et al., 1989; Money et al., 1992; Parasuraman \& Martin, 1994; Scinto et al., 1994). However, this conclusion requires verification from studies in which resource allocation is manipulated in the context of dual-task procedures. Further evidence would be provided by systematically manipulating sample-stimulus characteristics such as presentation frequency and duration in comparisons of AD patients' performance and normal performance, and in animal studies of cholinergic drugs (Parkes \& White, 2000).

Our reanalyses clearly indicate that rate of forgetting is not affected in AD patients in the later stages of the disease (Figure 2). Previous reviews have suggested that patients in the early stage of AD show faster rates of forgetting than do controls (McDonald \& Overmier, 1998). Our reanalyses of studies said to support this conclusion indicate, however, that there is no convincing evidence for differences in rate of forgetting. Apart from problems of experimental design, prior studies have been limited by measurement difficulties. First, in order to compare rates of forgetting, it is important that performance measures vary on a scale that is theoretically equal-interval (Loftus, 1978; Wixted, 1990). Second, rates of forgetting should be assessed in terms of the rate parameter of a fitted function instead of the interaction term in analysis of variance (White, 1985; Wixted, 1990). That is, forgetting functions with several retention intervals should be generated in order for one to be able to examine rate of forgetting.

Research with nonhuman animals provides strong evidence that the cholinergic system plays a role in the encoding of information (Blokland, 1996; Everitt \& Robbins, 1997; Fibiger, 1991). Further, the studies reviewed in this paper clearly suggest that cholinergic antagonists reduce initial discriminability, and that cholinergic agonists increase it, or at least reverse the effect of an antagonist such as scopolamine (Parkes \& White, 2000). This effect on attentional or encoding processes is apparent across a variety of different species, including humans. Encoding difficulty, as reflected in changes in initial discriminability, is also apparent for a variety of different manipulations, including pharmacological blockade, lesions to basal forebrain structures, and naturally occurring cholinergic degeneration in patients clinically diagnosed with AD. Further, the same psychological disruption has occurred for a variety of matching-to-sample procedures.

Consistent with conclusions from recent views (Blokland, 1996; Everitt \& Robbins, 1997), there is compelling evidence to suggest that at least one of the roles of the cholinergic system is the encoding of information. It is now becoming more appreciated, however, that the involvement of the cholinergic system in cognitive function is not as simple as was first believed. Other neurotransmitter systems may interact with the cholinergic system. It is therefore not surprising that there has been limited success in the development of drugs for the treatment of AD. From a behavioral perspective, improved experimental design that allows specific psychological processes to be identified, and clear interpretations of the processes affected by drug administration, may provide greater insight into the role of different neurotransmitter systems in cognitive function. 


\section{REFERENCES}

Adamson, C., Foster, T. M., \& McEwan, J. S. A. (2000). Delayed matching to sample: The effects of sample-set size on human performance. Behavioral Processes, 49, 149-161.

Alzheimer's Association (1996). Alzheimer's disease fact sheet. Chicago: Author.

Bäckman, L., Small, B. J., \& Fratiglioni, L. (2001). Stability of the preclinical episodic memory deficit in Alzheimer's disease. Brain, 124, 96-102.

BARTUS, R. T. (1978). Evidence for a direct cholinergic involvement in the scopolamine-induced amnesia in monkeys: Effects of concurrent administration of physostigmine and methlyphenidate with scopolamine. Pharmacology, Biochemistry \& Behavior, 9, 833-836.

Bartus, R. T., Dean, R. L., Beer, B., \& Lippa, A. S. (1982). The cholinergic hypothesis of geriatric memory dysfunction. Science, 217, 408417.

BARTus, R. T., \& Johnson, H. R. (1976). Short-term memory in Rhesus monkey: Disruption from the anti-cholinergic scopolamine. Pharmacology, Biochemistry \& Behavior, 5, 39-46.

Blokland, A. (1996). Acetylcholine: A neurotransmitter for learning and memory? Brain Research Reviews, 21, 285-300.

Bowen, D. M., \& DAVISON, A. N. (1986). Biochemical studies of nerve cells and energy metabolism in Alzheimer's disease. British Medical Bulletin, 42, 75-80.

BrAAK, H. \& BRAAK, E. (1991). Neuropathological staging of Alzheimerrelated changes. Acta Neuropathologica, 82, 239-259.

BrAAK, H., \& BRAAK, E. (1995). Staging of Alzheimer's disease-related neurofibrillary changes. Neurobiology of Aging, 16, 271-288.

Chen, P., Ratcliff, G., Belle, S. H., Cauley, J. A., DeKosky, S. T., \& GANGULI, M. (2000). Cognitive tests that best discriminate between presymptomatic $\mathrm{AD}$ and those who remain nondemented. Neurology, 55, 1847-1853.

CORKIN, S. (1998). Functional MRI for studying episodic memory in aging and Alzheimer's disease. Geriatrics, 53, 13-15.

Coyle, J. T., Price, D. L., \& DeLong, M. R. (1983). Alzheimer's disease: A disorder of cortical cholinergic innervation. Science, 219, 1184-1190.

Davison, M. C., \& Tustin, R. D. (1978). The relation between the generalized matching law and signal-detection theory. Journal of the Experimental Analysis of Behavior, 29, 331-336.

Dawson, G. R., Bentley, G., Draper, F., Rycroft, W., Iverson, S. D., \& Pagella, P. G. (1991). The behavioral effects of heptyl physostigmine, a new cholinesterase inhibitor, in tests of long-term and working memory in rodents. Pharmacology, Biochemistry \& Behavior, 39, 865-871.

Dawson, G. R., \& Iversen, S. D. (1993). The effects of novel cholinesterase inhibitors and selective muscarinic receptor agonists in tests of reference and working memory. Behavioural Brain Research, 57, 143-153.

de Toledo-Morrell,L., Goncharova, I., Dickerson, B., Wilson, R. S., \& Bennett, D. A. (2000). From healthy aging to early Alzheimer's disease: In vivo detection of entorhinal cortex atrophy. In H. E. Sharfman, M. F. Witter, \& R. Schwartz (Eds.), The parahippocampal region: Implications for neurological and psychiatric diseases (Annals of the New York Academy of Sciences, Vol. 911, pp. 240-253). New York: New York Academy of Sciences.

Drachman, D. A., \& LeavitT, J. L. (1974). Human memory and the cholinergic system: A relationship to aging? Archives of Neurology, 30, 113-121.

DunNeTt, S. B. (1985). Comparative effects of cholinergic drugs and lesions of nucleus basalis or fimbria-fornix on delayed matching in rats. Psychopharmacology, 87, 357-363.

DunnetT, S. B., \& Fibiger, H. C. (1993). Role of forebrain cholinergic systems in learning and memory: Relevance to the cognitive deficits of aging and Alzheimer's dementia. Progress in Brain Research, 98, 413-420.

Dunnett, S. B., Rogers, D. C., \& Jones, G. H. (1989). Effects of nucleus basalis magnocellularis lesions in rats on delayed matching and non-matching to position tasks. European Journal of Neuroscience, 1, 395-406.

EDhouse, W. V., \& White, K. G. (1988). Sources of proactive interference in animal memory. Journal of Experimental Psychology: Animal Behavior Processes, 14, 56-71.

Everitt, B. J., \& Robbins, T. W. (1997). Central cholinergic systems and cognition. Annual Review of Psychology, 48, 649-684.

FIBIGER, H. C. (1991). Cholinergic mechanisms in learning, memory and dementia: A review of recent evidence. Trends in Neurosciences, 14, 220-223.

Flicker, C., Ferris, S. H., Kalkstein, D., \& Serby, M. (1994). A doubleblind, placebo-controlled crossover study of Ganglioside GM1 treatment for Alzheimer's disease. American Journal of Psychiatry, 151, 126-129.

Flicker, C., Ferris, S. H., \& Reisberg, B. (1991). Mild cognitive impairment in the elderly: Predictors of dementia. Neurology, 41, 10061009.

Freed, D. M., \& Corkin, S. (1988). Rate of forgetting in H.M.: 6-month recognition. Behavioral Neuroscience, 102, 823-827.

Freed, D. M., Corkin, S., \& Cohen, N. J. (1987). Forgetting in H.M.: A second look. Neuropsychologia, 27, 325-339.

Grady, C. L., Grimes, A. M., Patronas, N., Sunderland, T., Foster, N. L., \& RAPOPORT, S. I. (1989). Divided attention, as measured by dichotic speech performance, in dementia of the Alzheimer type. Archives of Neurology, 46, 317-320.

Haist, F., Shimamura, A. P., \& Squire, L. R. (1992). On the relationship between recall and recognition memory. Journal of Experimental Psychology: Learning, Memory, \& Cognition, 18, 691-702.

Harper, D. N., \& White, K. G. (1997). Retroactive interference and rate of forgetting in delayed matching-to-sample performance. Animal Learning \& Behavior, 25, 158-164.

Hart, R. P., Kwentus, J. A., Harkins, S. W., \& Taylor, J. R. (1988). Rate of forgetting in mild Alzheimer's-type dementia. Brain \& Cognition, 7, 31-38.

Huppert, F. A., \& Piercy, M. (1978). Dissociation between learning and remembering in organic amnesia. Nature, 275, 317-318.

Huppert, F. A., \& Piercy, M. (1979). Normal and abnormal forgetting in organic amnesia: Effect of locus of lesion. Cortex, 15, 385-390.

ISAAC, C. L., \& MAYES, A. R. (1999). Rate of forgetting in amnesia: II. Recall and recognition of word lists at different levels of organization. Journal of Experimental Psychology: Learning, Memory, \& Cognition, 25, 963-977.

Kirk, R. C., White, K. G., \& McNaughton, N. (1988). Low-dose scopolamine affects discriminability but not rate of forgetting in delayed conditional discrimination. Psychopharmacology, 96, 541-546.

Kopelman, M. D. (1985). Rates of forgetting Alzheimer-type dementia and Korsakoff's syndrome. Neuropsychologia, 23, 623-638.

LofTUS, G. R. (1978). On interpretation of interactions. Memory \& Cognition, 6, 312-319.

LofTus, G. R. (1985). Evaluating forgetting curves. Journal of Experimental Psychology: Learning, Memory, \& Cognition, 11, 397-406.

LuCE, R. D. (1963). Detection and recognition. In R. D. Luce, R. R. Bush, \& E. Galanter (Eds.), Handbook of mathematical psychology (pp. 105189). New York: Wiley.

Macmillan, N. A., \& Creelman, C. D. (1991). Detection theory: A user's guide. New York: Cambridge University Press.

Mash, D. C., Flynn, D. D., \& PotTer, L. T. (1985). Loss of M2 muscarinic receptors in the cerebral cortex in Alzheimer's disease and experimental cholinergic denervation. Science, 228, 115-117.

McCarthy, D., \& White, K. G. (1987). Behavioral models of delayed detection and their application to memory. In M. L. Commons, J. Mazur, J. A. Nevin, \& H. C. Rachlin (Eds.), Quantitative analysis of behavior: Vol. 5. The effect of delay and intervening events on reinforcement value (pp. 29-54). New York: Erlbaum.

McDonald, M. P., \& Overmier, J. B. (1998). Present imperfect: A critical review of animal models of the mnemonic impairments in Alzheimer's disease. Neuroscience \& Biobehavioral Reviews, 22, 99120.

McKeE, R. D., \& Squire, L. R. (1992). Equivalent forgetting rates in 
long-term memory for diencephalic and medial temporal lobe amnesia. Journal of Neuroscience, 12, 3765-3772.

Medina, L., \& Reiner, A. (1994). Distribution of choline acetyltransferase immunoreactivity in the pigeon brain. Journal of Comparative Neurology, 342, 497-537.

Miyamoto, M., Takahashi, H., Kato, K., Hirai, K., Ishihara, Y., \& Goto, G. (1996). Effects of 3-[1-(Phenylmethyl)-4-Piperidinyl]1-(2,3,4,5-Tetrahydro-1H-1-Benzazepin-8-yl)-1-PropanoneFumarate (TAK-147), a novel acetylcholinesterase inhibitor, on impaired learning and memory in animal models. Journal of Pharmacology \& Experimental Therapeutics, 227, 1292-1304.

Money, E. A., Kirk, R. C., \& McNaughton, N. (1992). Alzheimer's dementia produces a loss of discriminability but no increase in rate of memory decay in delayed matching to sample. Neuropsychologia, 30, 133-143.

Mufsson, E. J., Chin, E. Y., Cochran, E. J., Beckett, L. A., Bennett, D. A., \& Kordower, J. H. (1999). Entorhinal cortex betaamyloid load in individuals with mild cognitive impairment. Experimental Neurology, 158, 469-490.

Parasuraman, R, \& Martin, A. (1994). Cognition in Alzheimer's disease: Disorders of attention and semantic knowledge. Current Opinions in Neurobiology, 4, 237-244.

Parkes, M., \& White, K. G. (2000). Glucose attenuation of memory impairments. Behavioral Neuroscience, 114, 307-319.

Penetar, D. M., \& McDonough, J. H. (1983). Effects of cholinergic drugs on delayed match-to-performance of Rhesus monkeys. Pharmacology, Biochemistry \& Behavior, 19, 963-967.

Perry, E. K. (1986). The cholinergic hypothesis - ten years on. British Medical Bulletin, 42, 63-69.

Perry, E. K., Tomlinson, B. E., Blessed, G., Bergmann, K., Gibson, P., \& Perry, R. H. (1978). Correlation of cholinergic abnormalities with senile plaques and mental test scores in dementia. British Medical Journal, 2, 1457-1459.

Robinson, J. K., Wenk, G. L., Wiley, R. G., Lappi, D. A., \& Crawley, J. N. (1996). ${ }^{192}$ IgG-Saporin immunotoxin and ibotenic acid lesions of nucleus basalis and medial septum produce comparable deficits on delayed nonmatching to position in rats. Psychobiology, 24, 179-186.

Rubin, D. C., \& Wenzel, A. E. (1996). One hundred years of forgetting: A quantitative description of retention. Psychological Review, 103, 734-760.

Ruske, A. C., Fisher, A., \& White, K. G. (1997). Attenuation of scopolamine-induced deficits in delayed-matching performance by a new muscarinic agonist. Psychobiology, 25, 313-320.

Ruske, A. C., \& White, K. G. (1999). Attenuation of scopolamineinduced deficits in pigeons' attention to delayed matching-to-sample cues by D-cycloserine. Psychobiology, 27, 507-512.

SAfEr, D. J., \& Allen, R. P. (1971). The central effects of scopolamine in man. Biological Psychiatry, 3, 347-355.

Sahakian, B. J., Morris, R. G., Evenden, J. L., Heald, A., Levey, R, Philpot, M., \& Robbins, T. W. (1988). A comparative study of visuospatial memory and learning in Alzheimer-type dementia and Parkinson's disease. Brain, 111, 695-718.
Sahgal, A., Galloway, P. H., McKeith, I. G., Lloyd, S., Cook, J. H., Ferrier, N. I., \& EDWARdSon, J. A. (1992). Matching-to-sample deficits in patients with senile dementias of the Alzheimer or Lewy body types. Archives of Neurology, 49, 1043-1046.

Savage, L. M., \& Parsons, J. (1997). The effects of delay interval, intertrial interval, amnestic drugs, and differential outcomes on matching-toposition in rats. Psychobiology, 25, 303-312.

Scinto, L. F., Daffner, K. R., Castro, L., Weintraub, S., Vavrik, M., \& Mesulam, M. M. (1994). Impairment of spatially directed attention in patients with probable Alzheimer's disease as measured by eye movements. Archives of Neurology, 51, 682-688.

Small, G. W. (1998). The pathogenesis of Alzheimer's disease. Journal of Clinical Psychiatry, 59, 7-14.

Squire, L. R, \& Zola-Morgan, S. (1991). The medial temporal lobe memory system. Science, 25, 1380-1386.

Teal, J. J., \& Evans, H. L. (1982). Effects of DDAVP, a vasopressin ana$\log$, on delayed matching behavior in the pigeon. Pharmacology, Biochemistry \& Behavior, 17, 1123-1127.

Volger, B. W. (1991). Alternatives in the treatment of memory loss in patients with Alzheimer's disease. Clinical Pharmacy, 10, 447-450.

White, K. G. (1985). Characteristics of forgetting functions. Journal of the Experimental Analysis of Behavior, 44, 15-34.

White, K. G. (1991). Psychophysics of direct remembering. In M. L. Commons, J. A. Nevin, \& M. C. Davison (Eds.), Signal detection: Mechanisms (pp. 221-237). Hillsdale, NJ: Erlbaum.

White, K. G. (2001). Forgetting functions. Animal Learning \& Behavior, 29, 193-207.

White, K. G., \& Alsop, B. L. (1993). Cognitionin birds. In A. Sahgal(Ed.), Behavioural neuroscience: A practical approach (pp. 137-147). Oxford: Oxford University Press.

White, K. G., \& Bunnell-McKenzie,J. (1985). Potentiation of delayed matching by variable delays. Animal Learning \& Behavior, 13, 397 402.

White, K. G., \& HARPer, D. (1996). Quantitative reanalysis of lesion effects on rate of forgetting in macaques. BehaviouralBrain Research, 74, 223-227.

White, K. G., Ruske, A. C., \& Colombo, M. (1996). Memory procedures, performance, and processes in pigeons. Cognitive Brain Research, 3, 309-317.

Whitehouse, P. J. (1986). Neuronal loss and neurotransmitter receptor alterations in Alzheimer's disease. In A. Fisher, I. Hanin, \& C. Lachman (Eds.), Alzheimer's and Parkinson's disease: Strategies for research and development (pp. 85-94). New York: Plenum.

WiXTED, J. T. (1990). Analyzing the empirical course of forgetting. Journal of Experimental Psychology: Learning, Memory, \& Cognition, 16, 927-935.

Wixted, J. T., \& Ebbesen, E. B. (1991). On the form of forgetting. Psychological Science, 6, 409-415.

(Manuscript received May 30, 2000; revision accepted for publication August 15, 2001.) 